\title{
Preface to the Special Issue on User Modeling in Ubiquitous Computing
}

\section{ANTHONY JAMESON and ANTONIO KRÜGER}

The field of user modeling has come up with many techniques for modeling and adapting to computer users, for example, to their preferences, goals, and intentions, as well as to their cognitive and affective states. Until relatively recently, these methods were restricted to desktop systems, in which the user's external context could largely be neglected. With the increasing ubiquity of mobile and embedded devices, it has become clear that in many cases the recognition and modeling of the user's external context is essential.

Coming from the other direction, ubiquitous computing has generated many approaches to recognizing and modeling a user's context, for example his or her location, physical environment, and social environment. But in most cases there has been no explicit modeling of users themselves. Recently, an increasing number of researchers in this area have taken into account the fact that the external context alone may not determine the most appropriate adaptation to the user. So they have worked on methods for recognizing and adapting to aspects of the user such as their activities, general interests, and current information needs.

We believe that the field of user modeling can contribute significantly to the enhancement of the effectiveness and usability of ubiquitous computing systems. In turn, the field of ubiquitous computing, by building the technological basis for mobile and migrating systems, is offering the user modeling community opportunities to apply their methods to novel types of systems, extending the methods themselves in the process. The major conferences in both fields - the series User Modeling, Intelligent User Interfaces, Ubicomp and Pervasive, have seen several invited talks, technical sessions, and workshops during recent years that can be seen as relevant to both user modeling and ubiquitous computing. The time seems to be ripe to bring these two communities even closer together - hence this special issue.

Although each article in the special issue represents a unique perspective, the articles can be divided roughly into two groups:

1. The three articles in the first group focus on methods that enable intelligent environments to capture and represent information about users and contexts so as to enable the environment to adapt to both. A common theme is the importance of enabling users to understand system decisions that may be based on a variety of user- and context-related information collected over time in different locations and situations. 
2. The two articles in the second group focus on methods for user-centered design and in-depth evaluation for mobile context-aware systems. Both articles describe museum applications that apply user modeling methods to adapt an audio presentation to the visitor's context and interests.

In the first article in the first group, David Carmichael, Judy Kay, and Robert Kummerfeld describe the Consistent Modeling of Users, Devices and Sensors in a Ubiquitous Computing Scenario. They underscore the need to support users in scrutinizing not only the content of their user models but also the underlying acquisition processes. The authors demonstrate their approach in the context of a system that enables users to gain information about sensors and services in their environment. Empirical tests are presented that show that the approach is highly scalable in terms of the collected data and the frequency of the requests.

The second article, by Keith Cheverst, Hee Eon Byun, Dan Fitton, Corina Sas, Chris Kray, and Nicolas Villar is entitled Exploring Issues of User Model Transparency and Proactive Behavior in an Office Environment Control System. Like the first article, this one elaborates on the issue of comprehensible ubiquitous computing systems. The authors describe an intelligent office control system that applies machine learning techniques to learn, on the basis of the user's behavior, rules that help a system to control with some degree of proactivity various devices in an office environment, such as fans and heaters. The representation of user preferences in terms of fuzzy decision trees is intended to increase the intuitive comprehensibility of the rules for users. Users are given opportunities to inspect the learned rules and the underlying history of their own actions and also to modify the rules. Like the articles in the second group, this article also shows the importance of iterative user testing in real environments.

Both of the first two articles stress the need to look at personalization and contextualisation at the same time. The third article in the first group, by Andreas Zimmermann, Marcus Specht, and Andreas Lorenz, provides a relevant software framework for Personalization and Context Management. This framework merges user models and context models to empower designers of context-aware applications to increase the user-adaptivity of these applications. The authors illustrate the usefulness of their framework with two application examples that concern an intelligent advertising board and a context-aware personalized museum guide, respectively.

The two articles in the second group look in greater depth at the application domain of museum applications that are both context-aware and user-adaptive. The first article, by Daniela Petrelli and Elena Not, is entitled User-Centered Design of Flexible Hypermedia for a Mobile Guide - Reflections on the the HyperAudio Experience. It reviews the development of the well-known HyperAudio system and generalizes the results to a user-centered design methodology for mobile and ubiquitous applications. The second article in this group, by Marek Hatala and Ron Wakkary, looks at Ontology-Based User Modeling in an Augmented Audio Reality System for Museums. It shows how in-depth user evaluation can inform the design 
of context-aware applications. Taken together, the two articles discuss many aspects of the design life cycle of a mobile guide, including early user involvement (emphasized by Petrelli and Not) and richly informative user evaluations (a topic to which Hatala and Wakkary make some novel contributions). Although methods like these are widely accepted in the field of human-computer interaction, especially where more conventional systems are involved, they still require more attention in connection with intelligent and user-adaptive systems.

This special issue owes a great deal to its hidden coauthors: the dedicated team of expert reviewers who wrote extensive, perceptive, and constructive comments on earlier versions of the articles that you now see before you. These final versions are the result of an extensive iterative process of communication between the authors, the reviewers, and the guest editors. We would also like to thank the valuable comments and suggestions of the editorial board, which have helped to achieve the high quality of this special issue. We hope that the articles will stimulate a similarly fruitful exchange of ideas among researchers in both of the fields of user modeling and ubiquitous computing.

\section{Authors' Vitae}

Anthony Jameson (http://dfki.de/ jameson) is a principal researcher at the German Research Institute for Artificial Intelligence (DFKI) and an adjunct professor of human-computer interaction at the International University in Germany.

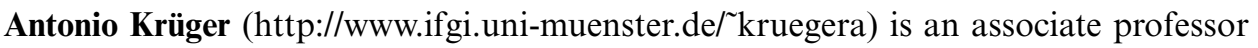
at the Institute for Geoinformatics and Computer Science of the University of Münster. He received his diploma and doctoral degree in Artificial Intelligence from Saarland University, Germany. Dr. Krüger is working in the areas of intelligent user interfaces, smart environments and mobile information systems. He is especially interested in the influence of ubiquitous computing on these fields, which motivated the realization of this special issue. He is also one of the co-founders of Eyeled Ltd, a company dedicated to the design of mobile and ubiquitous information systems. 